\title{
Otoacoustic Emissions Probe Placement Checking: A Step Forward for Noninvasive Intracranial Pressure Monitoring
}

\author{
Jeferson J. da Silva ${ }^{1,2}$, Gregory Gerenton ${ }^{2}$, Paul Avan ${ }^{1}$, Fabrice Giraudet ${ }^{1}$ \\ ${ }^{1}$ University Clermont Auvergne, Laboratory of Neurosensory Biophysics, UMR INSERM 1107 \\ 28 Place Henri Dunant, Clermont-Ferrand, France \\ jeferson.da_silva@etu.uca.fr; paul.avan@uca.fr; fabrice.giraudet@uca.fr \\ ${ }^{2}$ ECHODIA S.A.R.L \\ 35 rue du Pré la reine, Clermont-Ferrand, France \\ grgerenton@gmail.com
}

\section{Extended Abstract}

Background: Noninvasive monitoring of intracranial pressure (ICP) has been a research topic for decades, as the invasive monitoring is through an intraventricular or intraparenchymal catheter that involves risks such as malposition, hemorrhage or infection. The ICP monitoring by sound-evoked responses from the inner ear, have been reliably hinted at relative noninvasive measurements $[1,2]$. One type commonly analyzed in these methods is distortion product otoacoustic emissions (DPOAE). In most studies the main parameter is the DPOAE phase shift for different body tilt position, which changes the ICP $[3,4]$. Since this is a relative measurement, an individual baseline is required which should not be changed during or between measures. However, calibration methods are usually used to control the levels of sound stimuli only, but their phases remain sensitive to probe positioning and (even slight) variations in the ear's impedance [5]. For that reason, DPOAE phase shift becomes susceptible to baseline variation during the test and loses its reference if the probe is removed from the ear canal. The aim of this study is to determine a detection method of probe positioning and air leaks as phase calibration. This will lead to provide more reliable measurements and will also allow a long-term follow-up of patients by removing and repositioning the probe without changing the baseline.

Methods: Fourteen individuals (ages: 20-43 years old) were included in this study and all exams were performed inside a soundproof booth. For the DPOAE test the ECHODIA ELIOS was used with the stimulation frequencies $1000 \mathrm{~Hz}(\mathrm{~F} 1)$ and $1203 \mathrm{~Hz}(\mathrm{~F} 2)$ at $65 \mathrm{~dB}$ SPL and the distortion product was 2F1-F2 $(797 \mathrm{~Hz})$. The probe positioning and sealing analysis (PPSA) was done using software developed via MATLAB. For stimulation a chirp $(1-8 \mathrm{kHz})$ was sent by the OAE probe, and the response sound was recorded through its microphone for offline processing. The probe positioning was determined by the notch frequency in the FFT of the response and the leaks detection due to the signal level decreasing at low frequencies. A tilt table was used to induce a slight variation of the ICP at four positions $\left(70^{\circ}, 0^{\circ},-20^{\circ}\right.$ and again $\left.70^{\circ}\right)$, and at each position the PPSA response and 25 seconds of DPOAE were recorded. This procedure was performed twice, the first using foam eartip and the second with a rubber eartip. Subjects were separated into two groups: i) PPSA did not indicate problems in either of the two tests; ii) PPSA indicated a problem in at least one position in one of the two tests. The variation of the DPOAE phase was verified for each position of the body, with the phase values normalized to $0^{\circ}$ for the first position. The two groups were compared in relation to the difference of the mean phases for each of the positions between the two tests (foam and rubber eartip), and the standard deviation of the difference.

Data collection was performed, and we are in the final phase of data analysis.

Keywords: Intracranial pressure, Otoacoustic emissions, Signal processing, Health Monitoring Devices.

\section{References}

[1] F. Giraudet et al., "Noninvasive detection of alarming intracranial pressure changes by auditory monitoring in early management of brain injury: a prospective invasive versus noninvasive study," Crit. Care, vol. 21, no. 1, Mar. 2017.

[2] E. M. Bershad et al., "Intracranial pressure modulates distortion product otoacoustic emissions: A proof-of- principle study," Neurosurgery, vol. 75, no. 4, pp. 445-454, 2014. 
[3] A. R. Loiselle, E. de Kleine, P. van Dijk, and N. M. Jansonius, "Noninvasive intracranial pressure assessment using otoacoustic emissions: An application in glaucoma," PLoS One, vol. 13, no. 10, p. e0204939, Oct. 2018.

[4] B. Büki et al., "Middle-ear influence on otoacoustic emissions. II: Contributions of posture and intracranial pressure," Hear. Res., vol. 140, no. 1-2, pp. 202-211, 2000.

[5] P. Avan, B. Büki, B. Maat, M. Dordain, and H. P. Wit, "Middle ear influence on otoacoustic emissions. I: noninvasive investigation of the human transmission apparatus and comparison with model results.," Hear. Res., vol. 140, no. 12, pp. 189-201, Feb. 2000. 